INPLASY

PROTOCOL

To cite: Jin et al. Compound

Chinese medicine for radiation-induced lung injury: A protocol for systematic review and meta-analysis. Inplasy protocol 202230021. doi: 10.37766/inplasy2022.3.0021

Received: 05 March 2022

Published: 05 March 2022

Corresponding author: Yi Zhou

zhoumen1123456@163.com

Author Affiliation:

Chengdu University of

Traditional Chinese medicine.

Support: School support funds.

Review Stage at time of this submission: The review has not yet started.

Conflicts of interest: None declared.

\section{Compound Chinese medicine for radiation-induced lung injury: A protocol for systematic review and meta-analysis}

Jin, N1; Tu, S2; Xiong, P3; Zhang, Y4; Mo, J5; Zhou, Y6.

Review question / Objective: Radiation-induced lung injury (RILI) is a non-infectious inflammation caused by radiation damage to the normal lung tissue of patients. RILI is one of the common complications after radiotherapy for chest tumors, with the incidence of more than $30 \%$, which seriously affects the quality of life of patients and even endangers their lives. At present, the effective treatment of RILI is not comprehensive, western clinical treatment of glucocorticoids and anti-inflammatory drugs, the clinical efficacy is not ideal. Traditional Chinese medicine compound Chinese medicine has certain advantages in the intervention of RIUI treatment, which has attracted the attention of many researchers. However, the efficacy and safety of various TCM compounds in the treatment of RIUI have not been comprehensively evaluated.

Condition being studied: Radiation-induced lung injury.

INPLASY registration number: This protocol was registered with the International Platform of Registered Systematic Review and Meta-Analysis Protocols (INPLASY) on 05 March 2022 and was last updated on 05 March 2022 (registration number INPLASY202230021).

\section{INTRODUCTION}

Review question / Objective: Radiationinduced lung injury (RILI) is a noninfectious inflammation caused by radiation damage to the normal lung tissue of patients. RILI is one of the common complications after radiotherapy for chest tumors, with the incidence of more than
$30 \%$, which seriously affects the quality of life of patients and even endangers their lives. At present, the effective treatment of RILI is not comprehensive, western clinical treatment of glucocorticoids and antiinflammatory drugs, the clinical efficacy is not ideal. Traditional Chinese medicine compound Chinese medicine has certain advantages in the intervention of RIUI 
treatment, which has attracted the attention of many researchers. However, the efficacy and safety of various TCM compounds in the treatment of RIUI have not been comprehensively evaluated.

Condition being studied: Radiation-induced lung injury.

\section{METHODS}

Participant or population: Participants are aged 18 years or older and meet the diagnostic criteria classification of RILI in the Radiation Therapy Oncology Group, regardless of gender, race, nationality or education level.

Intervention: On the basis of the control group, compound Chinese medicine was given to treat. There is no limit to the dosage and duration of intervention.

Comparator: Placebo/no intervention (completion of conventional radiotherapy), or those treated with Western medicine received no intervention.

Study designs to be included: Randomized controlled trials.

Eligibility criteria: Randomized controlled trials of TCM compounding for radiation lung injury will be included. Nonrandomized controlled trials, observational studies, case reports, non-clinical studies, and other TCM interventions are excluded, with no language restrictions.

Information sources: The Cochrane Library, PubMed, EMBASE, SinoMed, CNKI, VIP, Web of Science, CBM and WANFANG database were systematically searched from inceptions until March 2022. Manually find references to relevant systematic reviews in this research area without language restrictions.

Main outcome(s): Number of cases of radiation pneumonia or radiation fibrosis during radiotherapy, and between 3 and 6 months after radiotherapy.
Additional outcome(s): 1. Acute radiological lung damage grading score (RTOG score) and physical functional status score (KPS score), and linear analogue selfassessment scale (LASA score) to compare pre- and post-treatment radiological lung damage rating, physical functional status, and quality of life. 2. Clinical signs and symptoms were observed. 3. Relevant clinical indicators.

Quality assessment / Risk of bias analysis: Two reviewers will assess the risk of bias using the cochrane collaboration tool. The content of the assessment includes: generation of random sequence (selection bias), allocation concealment (selection bias), blinding participants and personnel (performance bias), results blinding of evaluators (detection bias), incomplete result data(attrition bias), selective reporting(reporting bias)and other sources of bias, the risk of bias will be rated as "Iow risk", "high risk" or "unclear risk" in each area.Any inconsistencies will be determined in consultation with the third reviewer.

Strategy of data synthesis: Statistical analyses were conducted using Review Manager 5.3 software to assess the diversity of interventions and the potential heterogeneity between included studies. A random-effects model was used if the results showed low heterogeneity $(P \geq .1$ or $12 \leq 50 \%)$. When there was greater heterogeneity in clinical and compounded herbal medicines (P50\%), subgroup or sensitivity analyses were conducted to exclude sources of heterogeneity before combining the data.

Subgroup analysis: When results showed greater heterogeneity, subgroup analyses were conducted to explore the sources of heterogeneity based on elements such as age, gender, treatment modality, type of herbal compound, length of treatment or dose of medication.

Sensitivity analysis: When the risk of bias in the study was high, we performed a sensitivity analysis. The results were judged symmetrically by funnel plots, and 
after excluding abnormal results, the mate analysis was re-run to compare the differences between the two analyses to explore the robustness of the results.

Country(ies) involved: China.

Keywords: Radiation-induced lung injury, Compound Chinese medicine, systematic evaluation.

Contributions of each author:

Author 1 - Ni Jin.

Author 2 - Shijuan Tu.

Author 3 - Peiyu Xiong.

Author 4 - Yiyi Zhang.

Author 5 - Jingwen Mo.

Author 6 - Yi Zhou. 